OPEN ACCESS

Edited by:

Mingqiang $\mathrm{Li}$,

Sun Yat-Sen University, China

Reviewed by:

Liang Chen,

Fudan University, China

Run Zhang,

The University of Queensland,

Australia

${ }^{*}$ Correspondence:

Wei Liu

liuwei3359@pumch.cn

Xukun Zhou

zhouxukundoctor@sina.cn

tThese authors have contributed equally to this work

Specialty section:

This article was submitted to Biomaterials,

a section of the journal

Frontiers in Materials

Received: 20 May 2021

Accepted: 29 July 2021

Published: 14 September 2021

Citation:

Liu H, Zhuang F, Zhang C, Ai W, Liu W and Zhou $X$ (2021) Functionalized

Organic-Inorganic Liposome

Nanocomposites for the Effective

Photo-Thermal Therapy of

Breast Cancer.

Front. Mater. 8:710187.

doi: 10.3389/fmats.2021.710187

\section{Functionalized Organic-Inorganic Liposome Nanocomposites for the Effective Photo-Thermal Therapy of Breast Cancer}

\author{
Haibin Liu ${ }^{1,2+}$, Feng Zhuang ${ }^{1+}$, Chengjian Zhang ${ }^{1}$, Wanzhao Ai $^{1}$, Wei Liu ${ }^{1,3 *}$ and Xukun Zhou ${ }^{1 *}$ \\ ${ }^{1}$ Department of General Surgery, Hospital of Xinjiang Production and Construction Corps, Urumchi, China, ${ }^{2}$ Department of \\ Gastrointestinal Surgery, China-Japan Friendship Hospital, Beijing, China, ${ }^{3}$ Department of General Surgery, Peking Union \\ Medical College Hospital, Beijing, China
}

Organic-inorganic nanocomposites for photothermal therapy of cancers emerged as a promising strategy against malignant tumors. However, it is still a big challenge to develop a nanocomposite system that can maximize the synergistic photo-thermal therapy effect as well as preserve high stability for simultaneous delivery of the chemotherapeutic drugs and photo-thermal agents. Here, we have exploited an organic liposome containing inorganic core for co-loading the aggregates of bovine serum albumin (BSA), indocyanine green (ICG), and doxorubicin (DOX), abbreviated as BID-liposomal nanocomposites. The three kinds of substances were aggregated in the core of liposomal nanocomposites through hydrophobic and electrostatic interactions. In vitro characterization shows that BID-liposomal nanocomposites were spherical nanoparticles with size of $30-50 \mathrm{~nm}$ and good storage stability. Moreover, BID-Liposomal nanocomposites illustrate the strongest cytotoxicity among all the formulations against murine 4T1 tumor cells. In breast cancer-bearing mouse models, BID liposomes lead to significant improvements in tumor inhibition effects with no obvious toxicity. Therefore, the BID-liposomal nanoparticle is believed to be a promising strategy for chemo-photothermal therapy against cancers.

Keywords: functional materials, inorganic, photothermal therapy, liposome, anticancer

\section{INTRODUCTION}

As one of the standard-of-care cancer treatments, chemotherapy has been in a dilemma due to lack of tissue targeting and dose-limiting toxicities (Fan et al., 2017). With the rapid development of nanomedicine, scientists have developed many new therapeutic strategies against cancer, for example, photodynamic therapy (PDT), radiotherapy, gene therapy, immunotherapy, photothermal therapy (PTT) Min et al. (2017), Li et al. (2018a), Kedmi et al. (2018), Liu et al. (2019a), Chen et al. (2019), and cancer gas therapy (Opoku-Damoah et al., 2021). However, a monotherapy is not sufficient for effective cancer therapy. A great number of combination therapies has been reported, especially the combination of chemotherapy and photothermal therapy, as a promising strategy. Liu et al. (2021) developed a multifunctional nanoplatform which can realize $\mathrm{pH}$-sensitive drug release in an acidic tumor environment and the efficient photo-thermal conversion of ICG/Cu-LDH, causing synergism to significantly improve the therapeutic effect of anticancer. 
For photothermal therapy (PTT), the laser with a nearinfrared (NIR) light was applied to irradiate the photothermal agents (PTAs), such as ICG and Prussian blue, to achieve strong penetration into human tissues (Hu et al., 2017; Yang et al., 2017; Pan et al., 2018a; Pan et al., 2018b; Shan et al., 2018; Xue et al., 2018; Liu et al., 2019b; Chen et al., 2020a; Chen et al., 2020b). Most of the above agents are actively or passively carried and stored around the tumor through various targeted technologies. These photosensitizers could generate heat under NIR light. When the local temperature reaches above $40^{\circ} \mathrm{C}$, tumor cells will be inhibited or killed at high temperatures (Yang et al., 2017). In a wide range of photothermal agents, ICG is an FDA-approved reagent for in vivo imaging because of its good biocompatibility and NIR fluorescent characteristics. Besides the application in disease imaging, ICG is also widely applied in the area of photothermal therapy due to its strong absorption ability and a good photothermal conversion ability. However, it is limited by the defect of easy diffusion in vivo and repeated-irradiation causing photothermal conversion instability (Shan et al., 2018; Liu et al., 2019b).

Doxorubicin (DOX) is a classic and effective chemotherapy drug with a wide antitumor spectrum, which is often used in the treatment of malignant lymphoma, acute leukemia, breast cancer, gastric cancer, lung cancer, and other different types of cancer (Huang et al., 2015; Chen et al., 2017; Li et al., 2018b; Hao et al., 2018; Ni et al., 2018; Song et al., 2019). DOX has an excellent therapeutic effect and extensive application, but also has many untoward effects, such as severe side effects on the heart (Li et al., 2018b). For alleviation of toxicity, a version of the chemotherapy medication doxorubicin, Doxil, has been developed by a liposome-based strategy (Lyon et al., 2018; Penson et al., 2020). Liposomal nanocomposites are nanoscale particles nanoparticle delivery systems with enhanced permeability and retention (EPR) have unique advantages in cancer treatment. As a result, Doxil has a longer half-life in the blood circulation, leading to more time taken to reach the time tissue and penetrate the tumor tissue more easily and stay in the tumor tissue longer. Doxil has become a classic example for the delivery of chemo-drugs (He et al., 2019).

Here, we proposed to develop efficient chemo-photo-thermal composite systems of liposomal nanocomposites co-loading three components of ICG, DOX, and BSA. The ICG/DOX electrostatic interactions and stong binding capacity of ICG with BSA enable the aggregate formation of the above three substances. The aggregates is crucial for the photothermal effect of ICG molecules, sustain release of both ICG and DOX, and the stability of the whole systems. In this study, BID-liposomal nanocomposites were firstly prepared and characterized in vitro. Then, the cytoxicity and in vivo antitumor activity were investigated in various breast cancer cells and tumorbearing mouse models.

\section{MATERIALS AND METHODS}

\section{Materials}

$\mathrm{CaCl}_{2}, \mathrm{Na}_{2} \mathrm{HPO}_{4}$, cyclohexane, doxorubicin hydrochloride (DOX) were purchased from Aladdin Company; bovine serum albumin (BSA), indocyanine green (ICG), and IGEPAL ${ }^{\circledR}$ CO-520 were obtained from Sigma-Aldrich, Inc.; phospholipids (DOTAP, DOPA, and DSPE-PEG2000) were ordered from Avanti Polar Lipid, Inc. Other reagents were analytically pure. All reagents are applied directly without further purification. Deionised water was used in experiments.

4T1 cells (mouse breast cancer cells) were obtained from the American Type Culture Collection. Female NOD/SCID mice with 6-8 weeks of age were used for development of cancerbearing mouse models. All work on animals was approved by the Institutional Animal Care and Use Committee at the China-Japan Friendship Hospital.

\section{Preparation of BID-Liposomal Nanocomposites}

Based on previous research Li et al. (2012), Luo et al. (2016), the BIDliposomal nanocomposites were prepared by two steps including an inverse microemulsion method and a film dispersion method. First, $0.3 \mathrm{ml}$ of $500 \mathrm{mM} \mathrm{CaCl}_{2}$ solution and $6 \mathrm{mg} \mathrm{BSA} / \mathrm{ICG}(\mathrm{W} / \mathrm{W}=5 / 1)$ aggregates were dispersed into $15 \mathrm{ml}$ cyclohexane/IGEPAL ${ }^{\circledR} \mathrm{CO}$ 520 mixed solution under the condition of sonication to form a water-in-oil reverse microemulsion. At the same time, a similar microemulsion containing $0.3 \mathrm{ml}$ of $25 \mathrm{mM} \mathrm{Na}_{2} \mathrm{HPO}_{4}, 1 \mathrm{mg}$ DOX, and $0.2 \mathrm{ml}$ DOPA $(20 \mathrm{mg} / \mathrm{ml})$ was also prepared. Then, the two microemulsions above were mixed together to obtain calcium phosphate granules. After demulsification and centrifugation thrice, the calcium phosphate granules as a core were collected in chloroform for further use. Second, the lipid film was prepared using $1 \mathrm{ml}$ of calcium phosphate core, $0.1 \mathrm{ml}$ of DOTAP/cholesterol (1:1, $10 \mathrm{mM}$ ), and $0.1 \mathrm{ml}$ of DSPE-PEG2000 $(3 \mathrm{mM})$. All the materials were mixed in chloroform and subjected to rotatory evaporation to remove organic solvents. Finally, $1 \mathrm{ml}$ Tris- $\mathrm{HCl}$ buffer ( $\mathrm{pH} 7.4$ ) was added into the bottle with a lipid film. Under the condition of ultrasonic dispersion, BID-liposomal nanocomposites were obtained and further purified by dialysis (M.W. 3,500 KD) in water for $24 \mathrm{~h}$. The purified BID-liposomal nanocomposites were stored in dark place at $4^{\circ} \mathrm{C}$.

\section{In vitro Characterization of BID-Liposomal Nanocomposites}

Transmission electron microscopy (TEM, JEOL174 1200EX) was used to observe and photograph the morphology and size of BIDliposomal nanocomposites. For the preparation of TEM samples, BID-liposomal nanocomposites or the inorganic components of the core were dropped onto copper grids followed by negative staining with $1 \%$ uranium acetate, respectively. The hydrodynamic diameters of BID-liposomal nanocomposites were assayed by a dynamic light scattering method (PSS ZPW388-NICOMP Particle Sizing System) at room temperature. The particle sizes of BID-Liposomal nanocomposites and ICG-Liposomal nanocomposites during storage at $4^{\circ} \mathrm{C}$ were recorded to evaluate the formulation stability.

In order to study the stability of BID-liposomal nanocomposites in vivo, BID-liposomal nanocomposites were added to the medium containing $10 \%$ FBS to simulate the 
intracorporeal environment. The concentration of BID-liposomal nanocomposites was $160 \mu \mathrm{g} / \mathrm{ml}$ (calculated by ICG concentration). The samples were incubated in a cell incubator at $37^{\circ} \mathrm{C}$ for 3 days and compared with the BID-liposomal nanocomposites stored at $4^{\circ} \mathrm{C}$, and the particle size difference at different time points was compared.

\section{In vitro Fluorescence and UV-Vis Spectrum Assay of BID-Liposomal Nanocomposites}

To study the fluorescent properties, we scanned the fluorescence spectrum of BID-liposomal nanocomposites. Before scanning, the samples were diluted 30 folds by Tris- $\mathrm{HCl}$ buffer or methanol. For the investigation of DOX fluorescence in liposomal nanocomposites, the excitation wavelength was set as $480 \mathrm{~nm}$. The emission wavelength ranged from 500 to $900 \mathrm{~nm}$. For the investigation of ICG fluorescence in liposomal nanocomposites, the excitation wavelength was set as $780 \mathrm{~nm}$. The emission wavelength ranged from 790 to $900 \mathrm{~nm}$.

To calculate the encapsulation efficiency of DOX and ICG in BID-liposomal nanocomposites, the drug concentrations were assayed by ultraviolet and visible (UV-Vis) spectrophotometry. The standard curve of DOX and ICG is plotted for quantitative determination of drug concentrations in BID-liposomes, which were destroyed with 10 -fold methanol before detection. The UV scanning wavelength ranges of DOX and ICG were set from 300 to $900 \mathrm{~nm}$, respectively.

\section{In vitro the Photo-Thermal Transformation Characteristics Assay}

In order to evaluate the photothermal properties of BIDliposomal nanocomposites in 96-well plates, different concentrations $(5,10,20,40,80$, and $160 \mu \mathrm{g} / \mathrm{ml}$, calculated with ICG concentration) of BID-liposomal nanocomposites $(0.2 \mathrm{ml})$ were exposed to $808 \mathrm{~nm}$ laser at $1 \mathrm{~W} / \mathrm{cm}^{2}$ concentration. Sample temperatures at different time points were recorded by thermal infrared imaging system (FOTRIC).

To further investigate the photothermal conversion stability of BID-liposomal nanocomposites, the sample with $160 \mathrm{mg} / \mathrm{ml}$ BID-liposomal nanocomposites (calculated with ICG concentration) was exposed to five cycles of NIR radiation. After $3 \mathrm{~min}$ of each irradiation $\left(1 \mathrm{~W} / \mathrm{cm}^{2}, 808 \mathrm{~nm}\right)$, the sample was cooled to room temperature and then re-exposed to the laser. Monitor the sample temperature change and draw the temperature change curve after five cycles.

\section{In vitro Cytotoxicity Assays}

To assess the cytotoxicity of the BID-liposomal nanocomposites, the viability of the cultured cells was measured according to the standard protocol of a methyl thiazolyl tetrazolium (MTT) method. (Anishiya et al., 2020). 4T1 tumor cells (5,000 cells each well) were seeded in 96-well plates and cultured under the condition of $37^{\circ} \mathrm{C}$ and $5 \% \mathrm{CO}_{2}$. After $24 \mathrm{~h}$, free DOX and DOXliposomal nanocomposites with different DOX concentrations were added into each well. After 24-h incubation, the cell culture solutions were discarded followed by PBS rinse thrice. $20 \mu \mathrm{l}$ MTT solution ( $5 \mathrm{mg} / \mathrm{ml}, 0.5 \% \mathrm{MTT})$ was added to each well and subsequently incubated for $4 \mathrm{~h}$. Then, $150 \mu \mathrm{l}$ dimethyl sulfoxide was added to each well on a shaker at the speed of $20 \mathrm{rpm}$ for $10 \mathrm{~min}$. The cytotoxicity of DOX formulations was further assayed by enzyme-linked immunoassay to measure the absorption value at $490 \mathrm{~nm}$ wavelength.

Similarly, 4T1 tumor cells $(5,000$ cells each well) were incubated in 96-well plates for $24 \mathrm{~h}$ followed by treatment with different concentrations of ICG formulations $(1,5,10 \mu \mathrm{g} /$ $\mathrm{ml})$. Then, each well was exposed to $808 \mathrm{~nm}$ laser $\left(1 \mathrm{~W} / \mathrm{cm}^{2}\right)$ for $1 \mathrm{~min}$. The wells without NIR treatment were used as control. After 48-h incubation, the optical density (OD) values were determined for the evaluation of cytotoxicity.

\section{In vivo Pharmacodynamic Experiments}

Female NOD/SCID mice (6-8 weeks old) were subcutaneously inoculated with $4 \mathrm{~T} 1$ cells $\left(1^{\star} 10^{6}\right)$ in the left armpit, respectively. When the solid tumor size reached approximately $100-150 \mathrm{~mm}^{3}$, the tumor-bearing mice were randomly divided into six groups and received the following treatment schemes: 1) PBS; 2) DOX (2 mg/kg DOX); 3) DOX-liposomes (2 mg/kg DOX); 4) ICG $(2 \mathrm{mg} / \mathrm{kg}$ ICG), plus irradiation; 5) ICG-Liposomes $(2 \mathrm{mg} / \mathrm{kg}$ ICG), plus irradiation; and 6) BID-Liposomes (the dose was calculated by DOX with $2 \mathrm{mg} / \mathrm{kg}$ ), plus irradiation. Irradiation was performed at $24 \mathrm{~h}$ post administration. The first day of administration was recorded as day 0 , and the dose was repeated every other day for an overall 8 times during the treatment period. The body weight of each mouse was recorded till the experimental end. At day 14, all the mice were sacrificed by cervical dislocation. Tumors were excised and weighed.

\section{Statistical Analysis}

Quantitative data were indicated as means \pm standard deviation (SD). Either a Student's t-test or a one way analysis of variance (ANOVA) was performed to assess the experimental results. A $p$-value of less than 0.05 was regarded to have a statistically significant difference.

\section{RESULTS AND DISCUSSIONS}

\section{Development of BID-Liposomal Nanocomposites}

Due to a high water solubility of free ICG and DOX (in hydrochloride form), injection of each drug alone would cause diffusion in the whole body, probably limiting the chemo-photothermal combination effect on cancer therapy (Iimuro, 2017; Bönisch et al., 2019). Although ICG and DOX could be formed into aggregates through electrostatic attraction, these aggregates are unstable, especially in a complex environment in vivo (Figure 1).

Here, to make full use of the interactions of various molecules, the BSA/ICG/DOX aggregates were turned into the core of the BID-liposomal nanoparticle (Figure 2). Besides the core, this liposomal nanoparticle contains outer leaflet of lipid bi-layers. All the components are crucial for the performance of BID-liposomal nanocomposites in vitro and in vivo. First, the BSA/ICG/DOX aggregates would be beneficial to exert a higher photothermal 


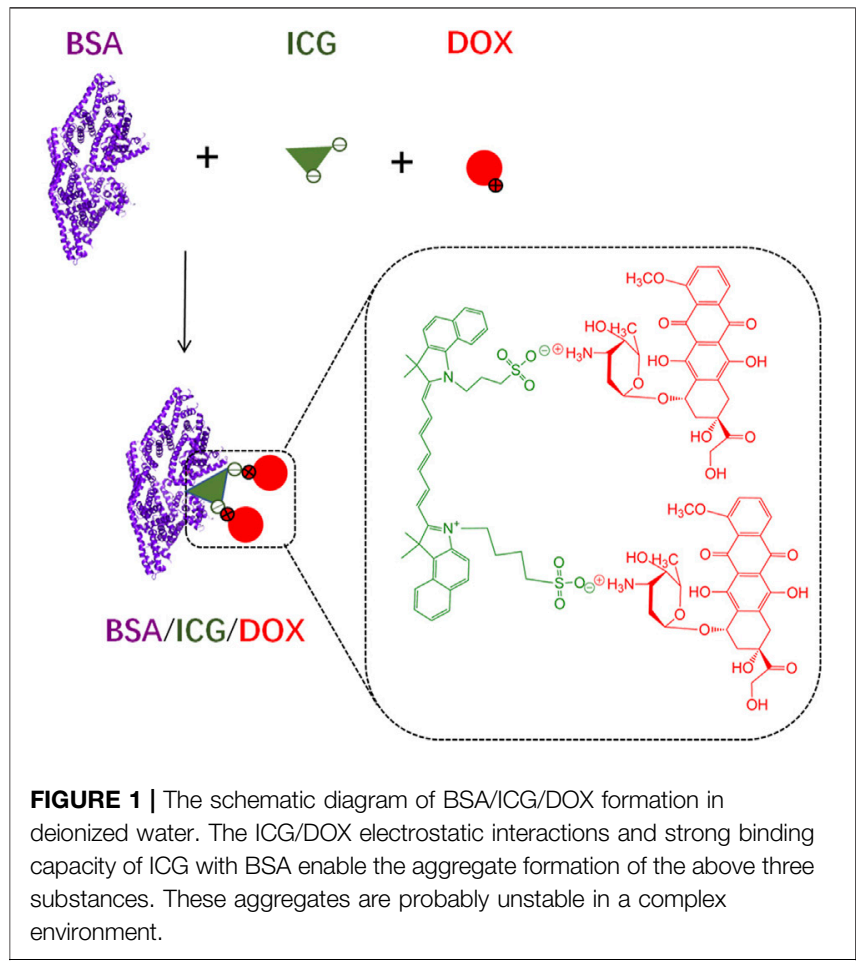

effect than free ICG. Second, the lipid coating with DSPE-PEG would be helpful for stable storage of BID-liposomal nanoparticle and long circulation after being injected. Third, these BIDliposomal nanocomposites could achieve controllable drug release in the acidic environment of tumor. Finally, under the condition of laser irradiation, the combination of DOX and ICG that co-delivered into the tumor site would significantly enhance the antitumor effect compared to either chemotherapy or photothermal alone.

It is noted that there are three kinds of nanostructures during the process of formulation: inorganic core without any lipid, inorganic core with a single layer of lipid (DOPA), and inorganic core with bilayer of lipid (named BID-liposomes in this study). The particle size of inorganic core without any lipid was extremely uncontrollable during the process of formulation. The inorganic core with a single layer of lipid has a uniform particle size but hydrophobic surfaces, difficult to be dissolved in water.

\section{In vitro Characterization of BID-Liposomal Nanocomposites}

To characterize the morphology and particle size of BIDliposomal nanocomposites, we observed the liposomal

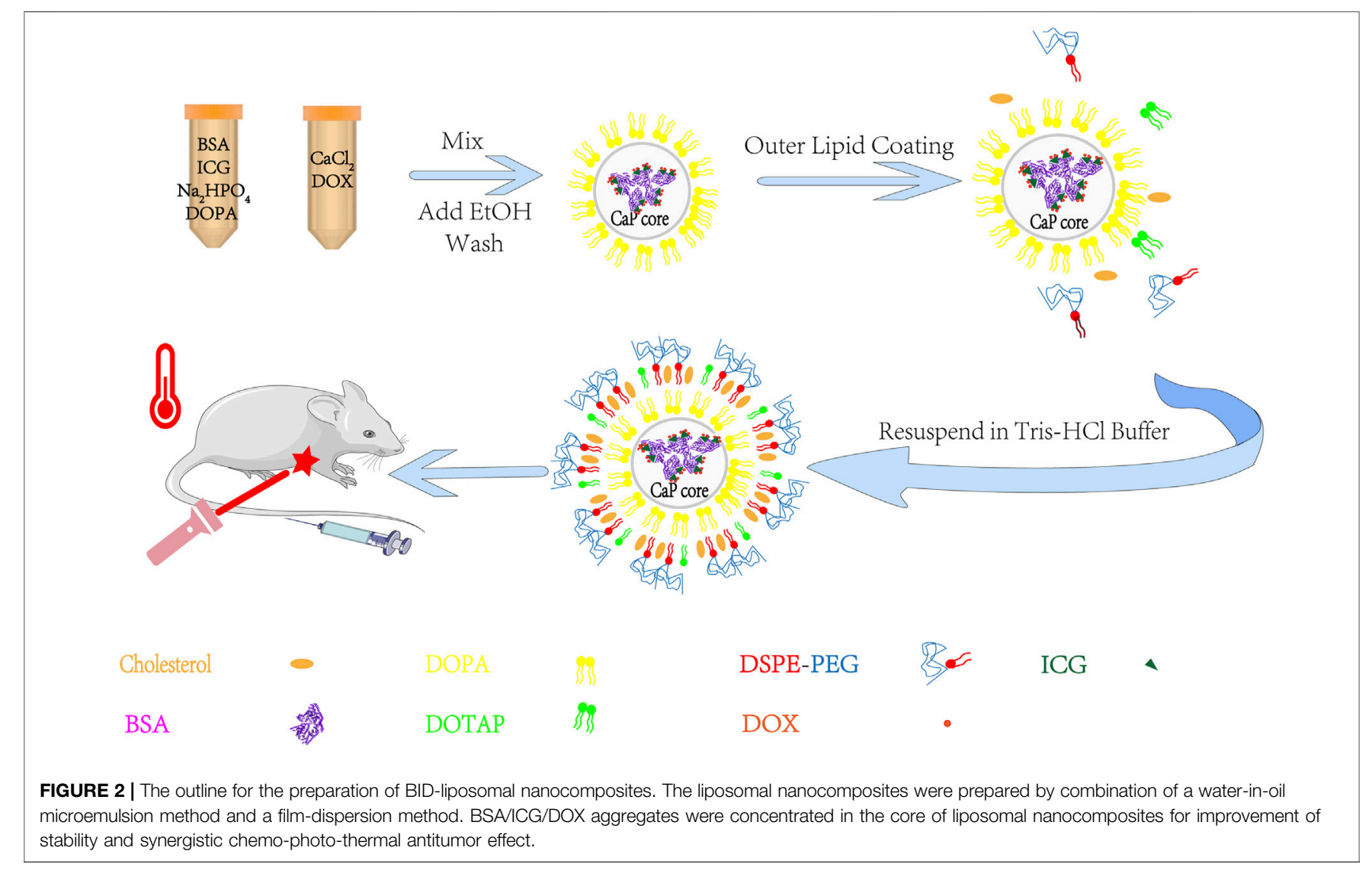



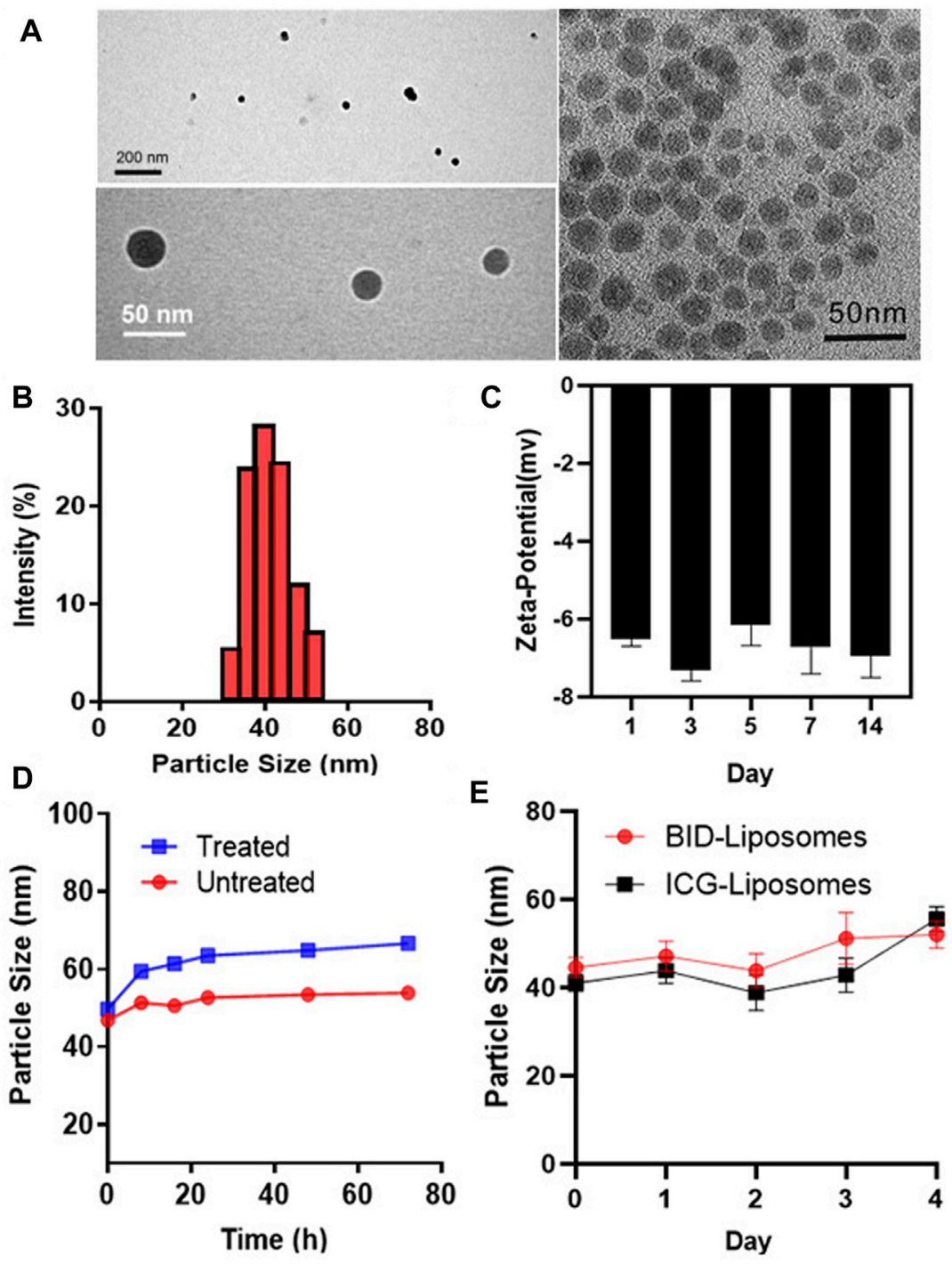

FIGURE 3 | In vitro characterization of BID-liposomal nanocomposites. (A) TEM images of BID-liposomal nanocomposites and the inorganic components of the core. Scale bar: 200 and $50 \mathrm{~nm}$. (B) Particle size assay of BID liposomal nanocomposites. (C) Changes in zeta potential of BID-liposomal nanocomposites over the incubation time. (D) Changes in the particle size of BID-liposomal nanocomposites treated or untreated with a medium containing $10 \%$ serum at $37^{\circ} \mathrm{C}$ within 3 days $\mathbf{( E )}$ Storage stablity of BID-liposomal nanocomposites and ICG-Liposomal nanocomposites at $4^{\circ} \mathrm{C}$.

nanocomposites under the transmission electron microscope (TEM) using a method of negative staining. TEM images show spherical solid cores with transparent shells, which is indicated as lipid bilayers (Figure 3A). As a control, inorganic core with a single layer of lipid was dissolved in the dichloromethane and imaged by TEM. The inorganic components of the core were the nano-sized calcium phosphate precipitates with a diameter of $20-30 \mathrm{~nm}$, which were generated by the reaction between $\mathrm{CaCl}_{2}$ and $\mathrm{NaHPO}_{4}$. The loose and porous structure of core can encapsulate the BSA/ICG/DOX aggregates. Due to these hydrophilic coatings, BID-liposomal nanocomposites could effectively avoid the uptake of reticuloendothelial system (RES) and thus achieve long circulation in blood. Moreover, the particle size of BID-Liposomal nanocomposites was determined by a dynamic light scattering (DLS) method (Figure 3B), in which the BIDliposomal nanocomposites were about $40 \mathrm{~nm}$, consistent with that in TEM images. Figure 3C showed that the changes in the zeta potential of the nanocomposites over the incubation time. The zeta potential could maintain relatively stable for at least 2 weeks. 

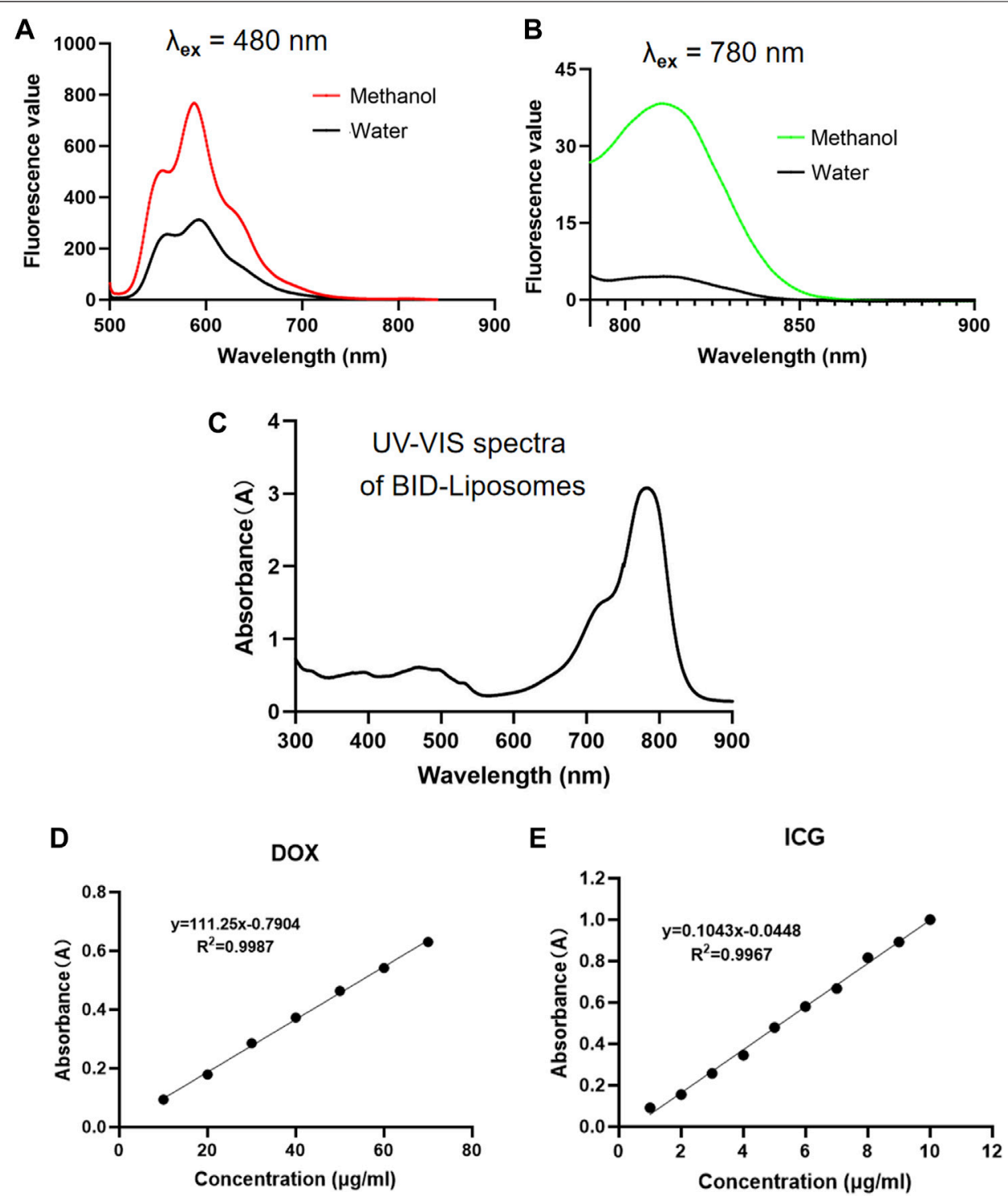

FIGURE 4 | In vitro fluorescence and UV-vis spectrum assay of BID-liposomal nanocomposites. (A)The emission spectra (500-900 nm) of BID-liposomes diluted with 30-fold methanol or water under the excitation wavelength of $480 \mathrm{~nm}$. (B) The emission spectra (790-900 nm) of BID-liposomes diluted with 30-fold methanol or water under the excitation wavelength of $780 \mathrm{~nm}$. (C) The UV-vis spectrum (300-900 nm) of BID liposomes. (D) Standard curve of DOX by the absorbance assay. (E) Standard curve of ICG by the absorbance assay.

The stability of BID-liposomal nanocomposites in vivo is an important prerequisite for their function. BID-liposomal nanocomposites incubated in a medium containing 10\% FBS for 3 days at $37^{\circ} \mathrm{C}$ were compared with the original sample. Figure 3D showed a slight increase in particle size of BIDliposomal nanocomposites. The adsorption of FBS on the periphery of BID-liposomal nanocomposites may lead to the increase of particle size.

To further study the storage stability of BID-liposomal nanocomposites which is one of the key parameters of nanoparticles, we determined the particle sizes at each preset time point and plotted the change of size as a function of time. Results showed that both BID-liposomal nanocomposites and
ICG-liposomal nanocomposites could maintain the same particle size (Figure 3E). This excellent stability is attributable to the small size of BID-liposomal nanocomposites and the protection of lipid bilayers.

\section{In vitro Fluorescence and UV-Vis Spectrum of BID-Liposomal Nanocomposites}

To characterize the fluorescent properties, we scanned the fluorescence spectra of BID-liposomal nanocomposites. For excitation of DOX in liposomal nanocomposites, a peak of emission wavelength at $590 \mathrm{~nm}$ was observed in either treated or untreated with methanol dilution of BID-liposomal 


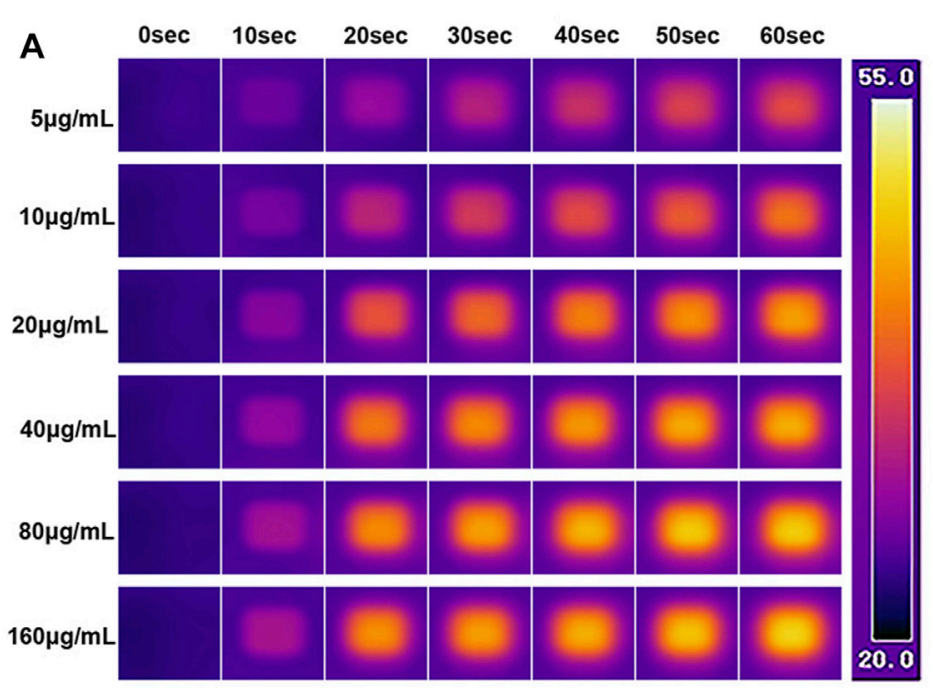

B

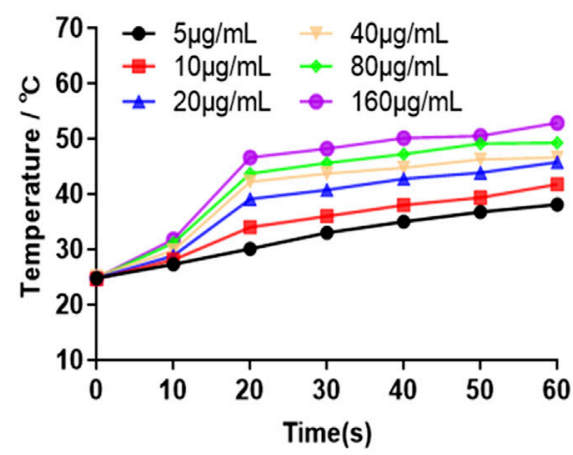

C

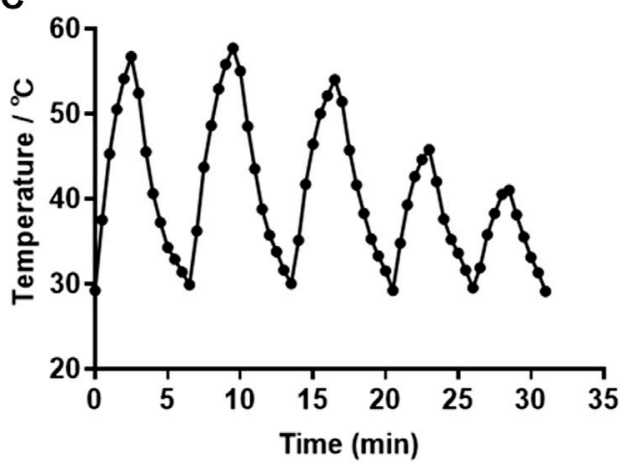

FIGURE 5 | The photothermal effect of the BID-liposomal nanocomposites. The thermographs (A) and corresponding heating curves (B) of the BID-liposomal nanocomposites at varied concentrations upon $808 \mathrm{~nm}$ laser exposure $\left(1 \mathrm{~W} / \mathrm{cm}^{2}\right)$ in 96 -well plates. BID-liposomal nanocomposites with different concentrations of ICG were assessed. (C) Heating curves of BID-liposomal nanocomposites subjected to repeated irradiation at $808 \mathrm{~nm}\left(1 \mathrm{~W} / \mathrm{cm}^{2}\right)$.

nanocomposites. (Figure 4A). For excitation of ICG in liposomal nanocomposites, both treated and untreated with methanol dilution of BID-liposomal nanocomposites illustrated obvious emission wavelength peak at $815 \mathrm{~nm}$ (Figure 4B).

The UV-vis absorbance spectra validated the successful encapsulation of ICG and DOX in BID-liposomal nanocomposites (Figure 4C). Based on the UV-vis spectra, the encapsulation efficiency of ICG and DOX in nanocomposites were $81.98 \pm 0.86 \%$ and $54.24 \pm 2.12 \%$, respectively, calculated by the standard curves of free ICG and DOX. All the above data suggest successful encapsulation of ICG and DOX in BID-liposomal nanocomposites due to the strong interaction among BSA, ICG, and DOX.

\section{In vitro Photo-Thermal Effect of BID-Liposomal Nanocomposites}

To assess the photo-thermal potency, the changes of temperature were monitored by using a thermal infrared imaging agent while the BID-liposomal nanocomposites were irradiated by a laser $\left(808 \mathrm{~nm}, 1 \mathrm{~W} / \mathrm{cm}^{2}\right)$ in vitro. Five different concentrations of BID-liposomal nanocomposites (5, 10, 20, 40, 80 , and $160 \mu \mathrm{g} / \mathrm{ml}$, calculated with ICG concentration) were used to research the temperature changes under NIR irradiation. After one minute irradiation, the temperature of each concentration of BID-liposomal nanocomposites increases sharply. As expected, there was a positive correlation between temperature and BID-liposomal nanocomposites concentration (Figure 5A). The $10 \mu \mathrm{g} / \mathrm{ml}$ nanoparticles could maintain the temperature above $40^{\circ} \mathrm{C}$ (Figure $5 \mathbf{B}$ ), which can produce a mild thermal effect, promoting the uptake of chemotherapeutic drugs by cells. Deionised water as blank control had no effect on NIR irradiation. The above data proved that nanoparticles have a good photothermal effect. The results showed that under NIR irradiation, BID-liposomal nanocomposites transform light into a high temperature that tumor cells cannot bear, thus inhibiting the growth of tumor cells or directly killing tumor cells.

ICG is the main component of photothermal effect in BIDliposomal nanocomposites. Although it has superior photo- 

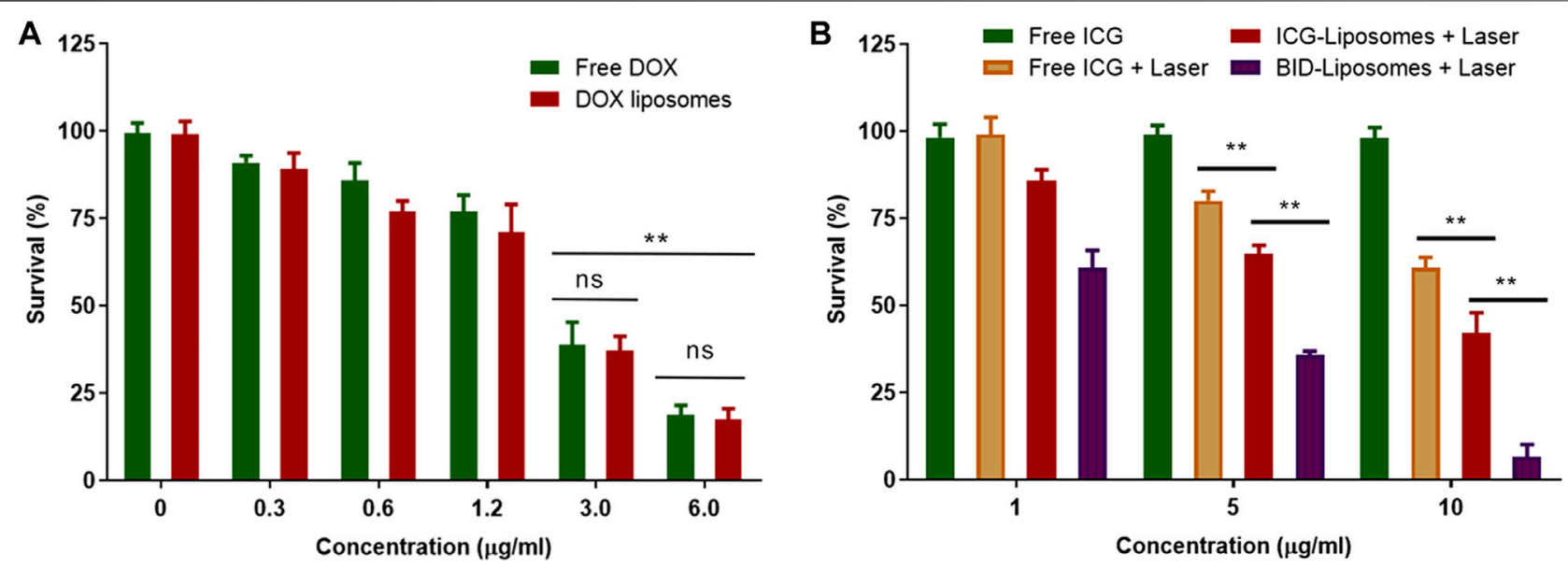

FIGURE 6 | Cytotoxicity assay against 4T1 breast cancer cells. (A) Survival percentage of 4T1 cells under the treatment of various concentrations of free DOX and DOX liposomal nanocomposites. (B) Survival percentage of $4 \mathrm{~T} 1$ cells under various treatments of chemicals or combined chemo-photo-thermal treatments (mean \pm SD; ${ }^{\star *} p<0.01$; ns, not significant; $n=3$ ).

thermal conversion performance, but there are still some problems, such as photobleaching and reduction of photothermal effects after repeated exposure to radiation. As shown in Figure 5C, BID-liposomal nanocomposites undergo repeated NIR irradiation. In the first three cycles, the photothermal effect of BID-liposomal nanocomposites kept good repeatability, but in the fourth cycle, the photothermal effect of BID-liposomal nanocomposites decreased. This means that the photothermal effect of the BID-liposomal nanocomposites will gradually decrease with the increase of radiation times, which is consistent with the results of previous studies (Shan et al., 2018; Liu et al., 2019b). However, BID-liposomal nanocomposites showed relatively stable photothermal production in the first three cycles, indicating their potential as photothermal therapy.

\section{In vitro Cytotoxicity Assays}

The cytotoxicity of BID-liposomal nanocomposites was evaluated in 4T1 cancer cells using an MTT assay. Prior to the determination of BID-liposomal nanocomposites-causing cytoxicity, the cell viability after treatment with free DOX and DOX-liposomal nanocomposites were assessed. In Figure 6A, both DOX and DOX-liposomal nanocomposites showed negligible toxicity against cells. When the concentration of DOX was up to $3 \mu \mathrm{g} / \mathrm{ml}$, obvious cell death in the cell culture well was observed under the microscopy. Overall, the BIDliposomal nanocomposites and free DOX illustrate comparable cytotoxicities, suggesting that liposomal materials were nontoxic. Theoretically, DOX concentration of DOX-liposomal nanocomposites could be enhanced by liposomal nanoparticlemediated cellular uptake but toxicity reduced by the sustained release of DOX from liposomal nanocomposites. Both of these factors eventually resulted in a similar drug concentration in cells and cytotoxicity of DOX-liposomal nanocomposites compared with free DOX.
As expected, free ICG without irradiation did not affect the survival of $4 \mathrm{~T} 1$ cells. After irradiation, the BID-liposomal nanocomposites showed the strongest cytotoxicity among all the formulations $(6.7 \pm 3.4 \%$ for BID-Liposomal nanocomposites, $42.1 \pm 5.8 \%$ for ICG-liposomal nanocomposites and $60.9 \pm 2.9 \%$ for free ICG, Figure 6B). This suggests that the ICG in aggregate form is beneficial to enhance the photo-thermal effect compared with free ICG. More importantly, ICG and DOX displayed a significant combination therapeutic effect against cancer cells (Zheng et al., 2013; Liu et al., 2019c). All these data suggest the necessity of encapsulating the ICG/DOX aggregates into the core of BID-liposomal nanocomposites.

Besides the direct tumor cell killing effect by photothermal therapy, more action mechanisms should be involved. According to previous reports, laser irradiation on ICG could induce ROS production and apoptosis of cancer cells, thus greatly enhanced the antitumor efficacy (Liu et al., 2021; Opoku-Damoah et al., 2021). The mechanisms of BID-liposomes will be further investigated in our future work.

\section{In vivo Antitumor Efficacy}

To further evaluate chemo-photo-thermal combination therapy effect against 4T1 breast cancer-bearing mouse models, we weighed the tumor weight of mice receiving various therapy regimes. In agreement with the cytotoxicity assay in vitro, it was clearly illustrated that BID-liposomes with irradiation showed the strongest inhibitory effects against 4T1 tumors among all the DOX formulations (Figure 7A). Also, free DOX, DOXLiposomes, free ICG plus laser, and ICG-liposomes plus laser showed anti-tumor efficacy to some extend. Under the laser irradiation, free ICG and ICG-liposomes led to a comparable effect, probably because of the strong binding capacity of ICG with albumin in vivo, causing formation of similar nanoparticle and EPR effect into tumor. All the above results demonstrate an 

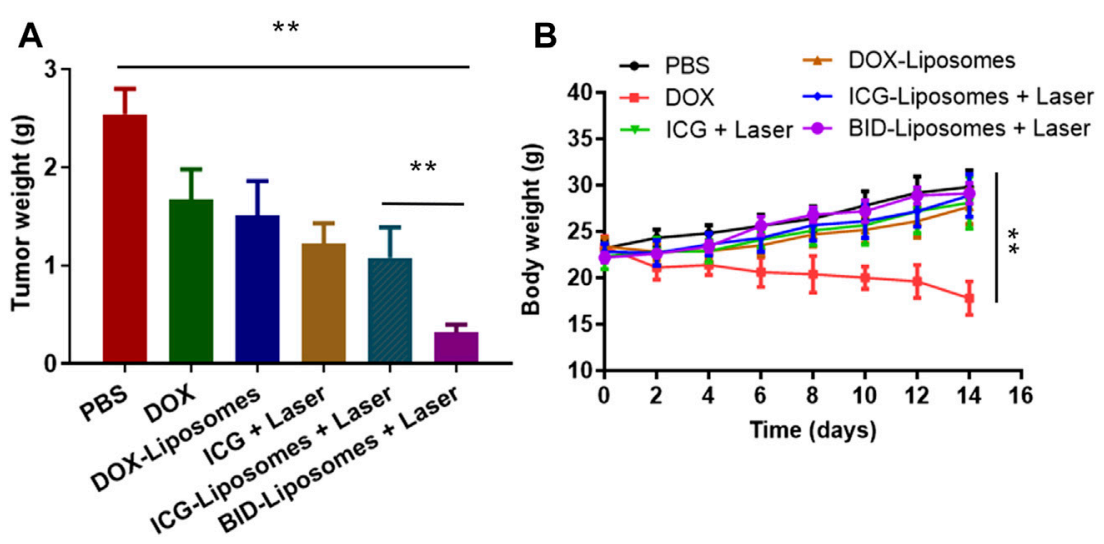

FIGURE 7 | In vivo chemo-photo-thermal therapy against 4T1 breast cancer-bearing mouse models. (A) Tumor weight of different groups of mice after treatments with various therapy regimes. (B) Body weight change curve of different mice. The number of mice in each group ( $n=6)$ (mean \pm SD; ${ }^{\star \star} p<0.01$; ns, not significant).

enhanced chemo-photo-thermal combination effect of BIDliposome.

During the antitumor experiment, the body weight of each mouse was also monitored after administration of various drugs. In Figure 7B, free DOX causes an obvious loss of mouse body weight, mainly due to the severe side effect of DOX, such as cardiotoxicity, myelosuppression, and so on. The DOXliposomes significantly reduces the side effects, probably due to the distribution change of liposome in vivo, avoiding the accumulation of DOX in the heart. Accordingly, all the other therapy regimes lead to a negligible change of body weight compared to phosphate buffer saline (PBS).

\section{CONCLUSION}

In this study, we have developed BID-liposomal nanocomposites that could simultaneously load three components of BSA, ICG, and DOX. The interactions among the drugs enable better formation of aggregates, accordingly resulting in multiple advantages of BID-liposomal nanocomposites. With the change of time, the BID-liposomal nanocomposites showed good physical stability without the precipitation and particle size increase of nanoparticles in vitro. As expected, the BIDliposomal nanocomposites showed excellent photothermal conversion under NIR irradiation and had certain stability under repeated irradiation. Moreover, BID-liposomal nanocomposites plus NIR laser irradiation show the strongest cytotoxicity among all the formulations. In breast cancer-bearing mouse models, BID-liposomes display significantly better tumor inhibition effects without obvious toxicity. Therefore, it is

\section{REFERENCES}

Anishiya, C. D. E., Rajendran, N. K., Jeyaraj, M., Ramu, A., and Rajan, M. (2020). Retinal Photoreceptors Targeting SA-G-AA Coated Multilamellar Liposomes concluded that the BID-liposomal nanocomposites holds great promise for chemo-photo-thermal therapy of malignant tumors.

\section{DATA AVAILABILITY STATEMENT}

The raw data supporting the conclusions of this article will be made available by the authors, without undue reservation.

\section{ETHICS STATEMENT}

The animal study was reviewed and approved by the Institutional Animal Care and Use Committee at Hospital of Xinjiang Production and Construction Corps.

\section{AUTHOR CONTRIBUTIONS}

$\mathrm{WL}$ and $\mathrm{XZ}$ are responsible for the research and design of the project. HL and FZ are responsible for specific test operation, data collection, analysis and processing, as well as manuscript writing. $\mathrm{CZ}$ and WA are responsible for literature collection, experimental assistance, and proofreading of manuscript.

\section{FUNDING}

This work was supported by the Foundation from Science and Technology Bureau of Xinjiang production and Construction Corps (2019BC006).

Carrier System for Cytotoxicity and Cellular Uptake Evaluation[J]. J. Liposome Res. 31 (2), 203-216. doi:10.1080/08982104.2020.1768111

Bönisch, N., Langan, E. A., and Terheyden, P. (2019). Kutanes Angiosarkom. Hautarzt 70 (9), 700-706. doi:10.1007/s00105-0194462-0 
Chen, J., Ding, J., Wang, Y., Cheng, J., Ji, S., Zhuang, X., et al. (2017). Sequentially Responsive Shell-Stacked Nanoparticles for Deep Penetration into Solid Tumors. Adv. Mater. 29 (32), 1701170. doi:10.1002/adma.201701170

Chen, Q., Chen, J., Yang, Z., Xu, J., Xu, L., Liang, C., et al. (2019). NanoparticleEnhanced Radiotherapy to Trigger Robust Cancer Immunotherapy. $A d v$. Mater. 31 (10), 1802228. doi:10.1002/adma.201802228

Chen, Y., Li, Z. H., Pan, P., Hu, J. J., Cheng, S. X., and Zhang, X. Z. (2020). TumorMicroenvironment-Triggered Ion Exchange of a Metal-Organic Framework Hybrid for Multimodal Imaging and Synergistic Therapy of Tumors. Adv. Mater. 32 (24), 2001452. doi:10.1002/adma.202001452

Chen, Y., Li, Z. H., Pan, P., Hu, J. J., Cheng, S. X., and Zhang, X. Z. (2020). TumorMicroenvironment-Triggered Ion Exchange of a Metal-Organic Framework Hybrid for Multimodal Imaging and Synergistic Therapy of Tumors. Adv. Mater. 32 (24), 2001452. doi:10.1002/adma.202001452

Fan, W., Yung, B., Huang, P., and Chen, X. (2017). Nanotechnology for Multimodal Synergistic Cancer Therapy. Chem. Rev. 117 (22), 13566-13638. doi:10.1021/acs.chemrev.7b00258

Hao, X., Li, C., Zhang, Y., Wang, H., Chen, G., Wang, M., et al. (2018). Programmable Chemotherapy and Immunotherapy against Breast Cancer Guided by Multiplexed Fluorescence Imaging in the Second Near-Infrared Window. Adv. Mater. 30 (51), 1804437. doi:10.1002/adma.201804437

He, H., Liu, L., Morin, E. E., Liu, M., and Schwendeman, A. (2019). Survey of Clinical Translation of Cancer Nanomedicines-Lessons Learned from Successes and Failures. Acc. Chem. Res. 52 (9), 2445-2461. doi:10.1021/ acs.accounts. 9 b00228

Hu, Y., Chi, C., Wang, S., Wang, L., Liang, P., Liu, F., et al. (2017). A Comparative Study of Clinical Intervention and Interventional Photothermal Therapy for Pancreatic Cancer. Adv. Mater. 29 (33), 1700448. doi:10.1002/adma.201700448

Huang, J., Shu, Q., Wang, L., Wu, H., Wang, A. Y., and Mao, H. (2015). Layer-bylayer Assembled Milk Protein Coated Magnetic Nanoparticle Enabled Oral Drug Delivery with High Stability in Stomach and Enzyme-Responsive Release in Small Intestine. Biomaterials 39, 105-113. doi:10.1016/ j.biomaterials.2014.10.059

Iimuro, Y. (2017). ICG Clearance Test and 99mTc-GSA SPECT/CT Fusion Images. Visc. Med. 33 (6), 449-454. doi:10.1159/000479046

Kedmi, R., Veiga, N., Ramishetti, S., Goldsmith, M., Rosenblum, D., Dammes, N., et al. (2018). A Modular Platform for Targeted RNAi Therapeutics. Nat. Nanotech 13 (3), 214-219. doi:10.1038/s41565-017-0043-5

Li, J., Yang, Y., and Huang, L. (2012). Calcium Phosphate Nanoparticles with an Asymmetric Lipid Bilayer Coating for siRNA Delivery to the Tumor. J. Controlled Release 158 (1), 108-114. doi:10.1016/j.jconrel.2011.10.020

Li, M., Sala, V., De Santis, M. C., Cimino, J., Cappello, P., Pianca, N., et al. (2018). Phosphoinositide 3-Kinase Gamma Inhibition Protects from Anthracycline Cardiotoxicity and Reduces Tumor Growth. Circulation 138 (7), 696-711. doi:10.1161/circulationaha.117.030352

Li, X., Lee, S., and Yoon, J. (2018). Supramolecular Photosensitizers Rejuvenate Photodynamic Therapy. Chem. Soc. Rev. 47 (4), 1174-1188. doi:10.1039/ c7cs00594f

Liu, C., Zhang, S., Li, J., Wei, J., Müllen, K., and Yin, M. (2019). A Water-Soluble, NIR-Absorbing Quaterrylenediimide Chromophore for Photoacoustic Imaging and Efficient Photothermal Cancer Therapy. Angew. Chem. Int. Ed. 58 (6), 1638-1642. doi:10.1002/anie.201810541

Liu, J., Sun, L., Li, L., Zhang, R., and Xu, Z. P. (2021). Synergistic Cancer Photochemotherapy via Layered Double Hydroxide-Based Trimodal Nanomedicine at Very Low Therapeutic Doses. ACS Appl. Mater. Inter. 13 (6), 7115-7126. doi:10.1021/acsami.0c23143

Liu, X., Wang, C., Ma, H., Yu, F., Hu, F., and Yuan, H. (2019). Water-Responsive Hybrid Nanoparticles Codelivering ICG and DOX Effectively Treat Breast Cancer via Hyperthermia-aided DOX Functionality and Drug Penetration. Adv. Healthc. Mater. 8 (8), 1801486. doi:10.1002/adhm.201801486

Liu, Y., Bhattarai, P., Dai, Z., and Chen, X. (2019). Photothermal Therapy and Photoacoustic Imaging via Nanotheranostics in Fighting Cancer. Chem. Soc. Rev. 48 (7), 2053-2108. doi:10.1039/c8cs00618k

Luo, C., Miao, L., Zhao, Y., Musetti, S., Wang, Y., Shi, K., et al. (2016). A Novel Cationic Lipid with Intrinsic Antitumor Activity to Facilitate Gene Therapy of TRAIL DNA. Biomaterials 102, 239-248. doi:10.1016/ j.biomaterials.2016.06.030
Lyon, P. C., Gray, M. D., Mannaris, C., Folkes, L. K., Stratford, M., Campo, L., et al. (2018). Safety and Feasibility of Ultrasound-Triggered Targeted Drug Delivery of Doxorubicin from Thermosensitive Liposomes in Liver Tumours (TARDOX): a single-centre, Open-Label, Phase 1 Trial. Lancet Oncol. 19 (8), 1027-1039. doi:10.1016/s1470-2045(18)30332-2

Min, Y., Roche, K. C., Tian, S., Eblan, M. J., McKinnon, K. P., Caster, J. M., et al. (2017). Antigen-capturing Nanoparticles Improve the Abscopal Effect and Cancer Immunotherapy. Nat. Nanotech 12 (9), 877-882. doi:10.1038/ nnano.2017.113

Ni, Q., Zhang, F., Zhang, Y., Zhu, G., Wang, Z., Teng, Z., et al. (2018). In Situ shRNA Synthesis on DNA-Polylactide Nanoparticles to Treat Multidrug Resistant Breast Cancer. Adv. Mater. 30 (10), 1705737. doi:10.1002/ adma.201705737

Opoku-Damoah, Y., Zhang, R., Ta, H. T., Amilan Jose, D., Sakla, R., and Xu, Z. P. (2021). Lipid-encapsulated Upconversion Nanoparticle for Near-Infrared Light-Mediated Carbon Monoxide Release for Cancer Gas Therapy. Eur. J. Pharmaceutics Biopharmaceutics 158, 211-221. doi:10.1016/ j.ejpb.2020.11.014

Pan, J., Wang, Y., Zhang, C., Wang, X., Wang, H., Wang, J., et al. (2018). AntigenDirected Fabrication of a Multifunctional Nanovaccine with Ultrahigh Antigen Loading Efficiency for Tumor Photothermal-Immunotherapy. Adv. Mater. 30 (8), 1704408. doi:10.1002/adma.201704408

Pan, J., Wang, Y., Zhang, C., Wang, X., Wang, H., Wang, J., et al. (2018). AntigenDirected Fabrication of a Multifunctional Nanovaccine with Ultrahigh Antigen Loading Efficiency for Tumor Photothermal-Immunotherapy. Adv. Mater. 30 (8), 1704408. doi:10.1002/adma.201704408

Penson, R. T., Valencia, R. V., Cibula, D., Colombo, N., Leath, C. A., Bidziński, M., et al. (2020). Olaparib versus Nonplatinum Chemotherapy in Patients with Platinum-Sensitive Relapsed Ovarian Cancer and a Germline BRCA1/2 Mutation (SOLO3): A Randomized Phase III Trial. J. Clin. Oncol. 38 (11), 1164-1174. doi:10.1200/JCO.19.02745

Shan, W., Chen, R., Zhang, Q., Zhao, J., Chen, B., Zhou, X., et al. (2018). Improved Stable Indocyanine Green (ICG)-Mediated Cancer Optotheranostics with Naturalized Hepatitis B Core Particles. Adv. Mater. 30 (28), 1707567. doi:10.1002/adma.201707567

Song, Q., Zheng, C., Jia, J., Zhao, H., Feng, Q., Zhang, H., et al. (2019). A Probiotic Spore-Based Oral Autonomous Nanoparticles Generator for Cancer Therapy. Adv. Mater. 31 (43), 1903793. doi:10.1002/adma.201903793

Xue, P., Yang, R., Sun, L., Li, Q., Zhang, L., Xu, Z., et al. (2018). Indocyanine GreenConjugated Magnetic Prussian Blue Nanoparticles for Synchronous photothermal/Photodynamic Tumor Therapy[J]. Nano-Micro Lett. 10 (4), 74. doi:10.1007/s40820-018-0227-Z

Yang, Y., Zhu, W., Dong, Z., Chao, Y., Xu, L., Chen, M., et al. (2017). 1D Coordination Polymer Nanofibers for Low-Temperature Photothermal Therapy. Adv. Mater. 29 (40), 1703588. doi:10.1002/adma.201703588

Zheng, M., Yue, C., Ma, Y., Gong, P., Zhao, P., Zheng, C., et al. (2013). Single-Step Assembly of DOX/ICG Loaded Lipid-Polymer Nanoparticles for Highly Effective Chemo-Photothermal Combination Therapy. ACS Nano 7 (3), 2056-2067. doi:10.1021/nn400334y

Conflict of Interest: The authors declare that the research was conducted in the absence of any commercial or financial relationships that could be construed as a potential conflict of interest.

Publisher's Note: All claims expressed in this article are solely those of the authors and do not necessarily represent those of their affiliated organizations, those of the publisher, the editors, and the reviewers. Any product that may be evaluated in this article, or claim that may be made by its manufacturer, is not guaranteed or endorsed by the publisher.

Copyright $\odot 2021$ Liu, Zhuang, Zhang, Ai, Liu and Zhou. This is an open-access article distributed under the terms of the Creative Commons Attribution License (CC $B Y)$. The use, distribution or reproduction in other forums is permitted, provided the original author(s) and the copyright owner(s) are credited and that the original publication in this journal is cited, in accordance with accepted academic practice. No use, distribution or reproduction is permitted which does not comply with these terms. 